\title{
Duodenal bile examination in identifying potential non-responders to bile salt treatment and its comparison with gall bladder bile examination
}

\author{
D K Agarwal, G Choudhuri, V A Saraswat, T S Negi, V K Kapoor, R Saxena
}

\begin{abstract}
The results of bile salt treatment in patients with radiolucent stones and a functioning gall bladder have been poor. In 42 of these patients awaiting cholecystectomy we determined the value of duodenal bile examination in predicting gall stone composition, and thus identifying those less likely to respond to bile salt therapy. Based on chemical analysis and scanning electron microscopy, 28 of $42(67 \%)$ gall stones retrieved at surgery were potentially insoluble. Microscopic examination of duodenal bile correctly identified $21(75 \%)$ of them: it predicted all four (100\%) pigment stones, three of six $(50 \%)$ calcium carbonate containing cholesterol stones, and 14 of 18 (78\%) cholesterol stones with pigment shells. It was nearly as reliable as microscopic examination of bile aspirated directly from the gall bladder during surgery (21 (75\%) $v 23(82 \%)$; $\mathrm{p}=\mathrm{NS}$ ). Furthermore, the presence of cholesterol crystals in duodenal bile was a more sensitive indicator than chemical detection of supersaturation (34 of $38(89 \%) v 25$ of 35 $(71 \%) ; p<0.05)$ for prediction of cholesterol gall stones. Microscopic examination of duodenal bile, if used as a screening test, could help to exclude potential non-responders and thereby improve considerably the results of oral bile salt treatment for gall stone dissolution. (Gut 1994; 35: 112-116)
\end{abstract}

Gall stone solvents currently available for clinical use are effective in dissolving only pure cholesterol stones. Bile salt treatment is unlikely to succeed if the stones are pigment stones or cholesterol stones with either sufficient amounts of inorganic calcium compounds or with an amorphous pigment layer on the outer surface. ${ }^{1-3}$ The limitations of current tests to identify potential non-responders while selecting patients for litholytic treatment account for the high failure rates of 70 to $87 \% .^{4-8}$ Conventional imaging methods like plain radiographs and oral cholecystograms have not been very useful. ${ }^{9-11}$ Lucency on plain radiograph of the abdomen and oral cholecystography are often misleading. ${ }^{10}{ }^{11}$ Several workers have recently evaluated the role of computed tomography for this purpose. ${ }^{12-14}$

Examination of gall bladder bile has been claimed to be a sensitive and reliable test for this purpose. However, gall bladder bile samples thus studied are obtained during cholecystectomy or by direct percutaneous puncture which restricts the diagnostic utility and clinical application of this method as a screening test for patients who wish non-invasive treatment for gall stones. ${ }^{15-18}$

Duodenal bile can easily be obtained by nasoduodenal intubation and can be examined for biliary microcrystals and the cholesterol saturation index (CSI). It is, however, considered to be dilute compared with gall bladder bile because of contamination with gastric, pancreatic, and intestinal secretions. The utility of duodenal bile examination in predicting gall stone composition and in identifying potential non-responders to bile salt treatment has not been prospectively evaluated in a sizeable group of subjects. ${ }^{16-18}$

We undertook this prospective study to evaluate the utility of duodenal bile examination in predicting gall stone composition and in identifying patients in whom bile salt treatment is less likely to succeed. We also wished to compare duodenal bile examination with bile aspirated directly from the gall bladder during surgery in terms of sensitivity to microscopic or chemical examination.

\section{Patients and methods}

Fifty four consecutive patients with symptomatic cholelithiasis who fulfilled with conventional inclusion criteria for oral bile acid treatment were studied. The gall bladder of each patient was ascertained to contain radiolucent gall stones and opacify on a 12 tablet oral cholecystogram. Pregnancy was excluded by investigations wherever indicated. Duodenal and gall bladder bile samples of each patient were subjected to microscopic and chemical examination and correlated with the composition of gall stones retrieved at cholecystectomy. Informed consent was obtained from each patient. The study protocol was approved by the institutional ethics committee.

Of the 54 patients who entered the study and had their duodenal bile examined, 12 were excluded due to failure to obtain gall bladder bile and gall stones (six did not report for elective surgery; six were operated on elsewhere). Data were complete in the remaining 42 patients: nine men and 33 women; age mean (SD) 38.9 (11.3) years, range 25 to 58 years.

\section{DUODENAL BILE}

After an overnight fast, nasoduodenal intubation was performed under fluoroscopy with BilbaoDotter tube and bile was collected after intravenous infusion of $5 \mu \mathrm{g}$ ceruletid (Takus ' $R$ ', Farmatalia Carlos Erba, Germany). Dark, thick viscous ' $B$ ' bile ${ }^{19}$ samples were pooled and mixed well and then collected in three aliquots. One 


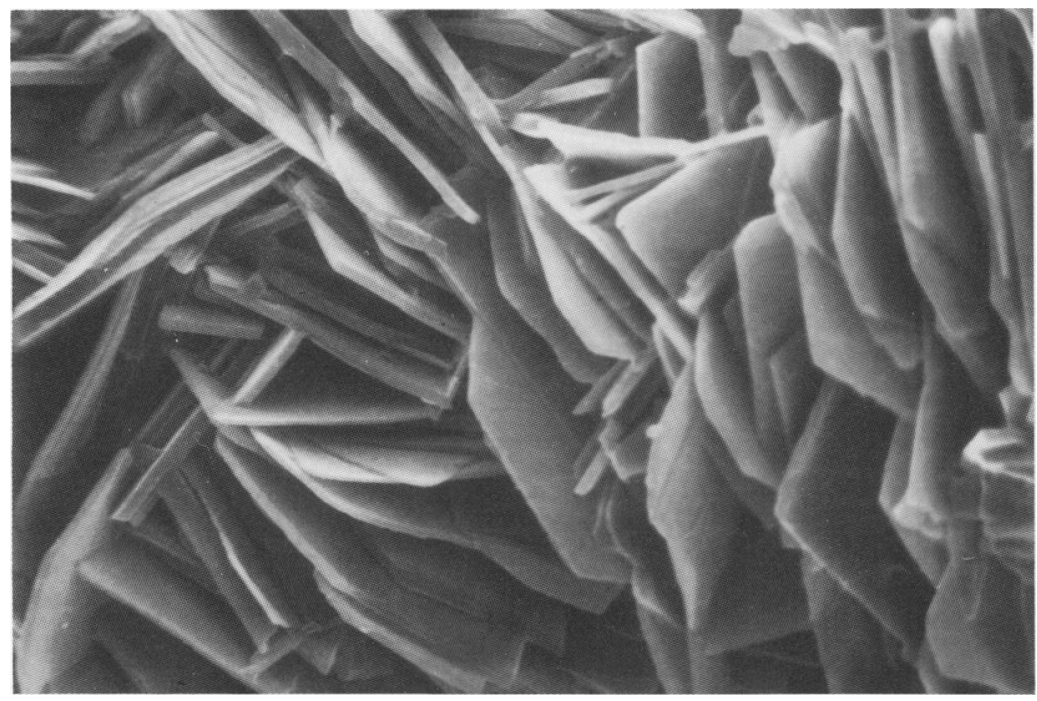

Figure 1: Scanning electron microphotograph (original magnification $\times 2000$ ) showing clear cholesterol crystals on the gall stone surface.

aliquot was examined within 30 minutes under the polarising microscope, the second was stored at $37^{\circ} \mathrm{C}$ for 24 hours for repeat microscopic examination if required, and the third aliquot was stored in a sealed glass vial at $-20^{\circ} \mathrm{C}$ for chemical examination.

\section{GALL BLADDER BILE AND GALL STONES}

During laparotomy, before removal of the gall bladder, the cystic duct was clamped and the gall bladder bile aspirated with a needle and syringe. While obtaining the bile sample, precaution was taken to ensure complete aspiration to avoid the effect of layering. Bile was collected in three aliquots and processed as described above for duodenal bile. The gall stones retrieved at surgery were washed with distilled water, dried, and stored at room temperature.

\section{MICROSCOPIC ANALYSIS OF BILE}

Altogether $2.5 \mathrm{ml}$ of bile were centrifuged at $2000 \mathrm{rpm}$ for 10 minutes; the supernatant was

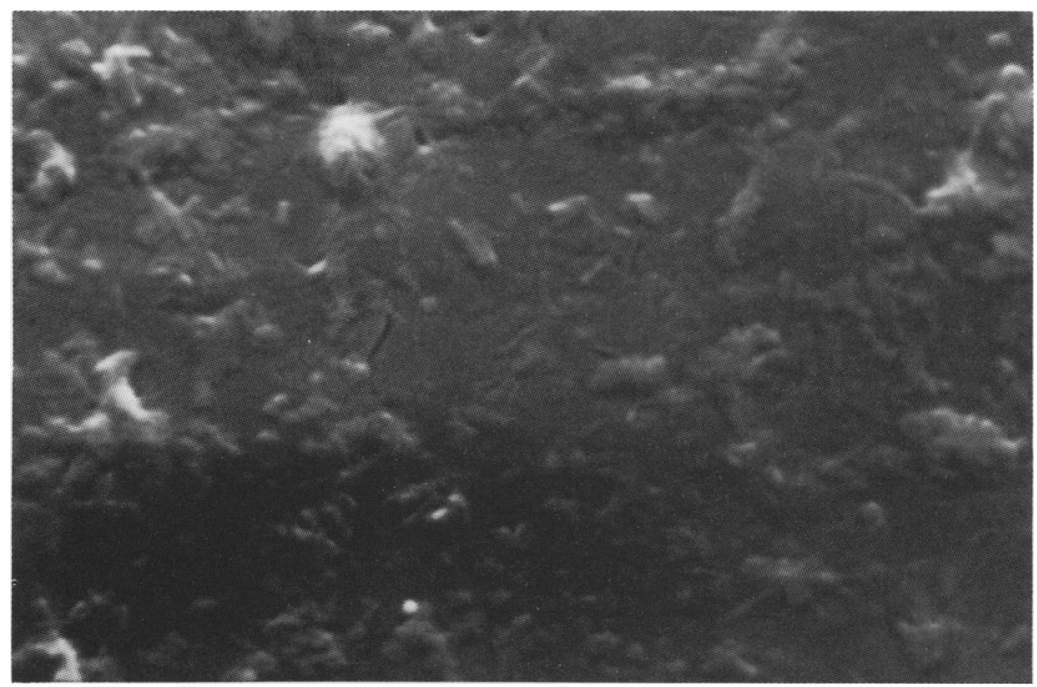

Figure 2: Scanning electron microphotograph (original magnification $\times 2000$ ) showing amorphous (pigment) shell covering the cholesterol gall stone surface. discarded and the sediment examined on a glass slide under the polarising microscope. The number of cholesterol monohydrate crystals, calcium bilirubinate granules, and microspheroliths of calcium carbonate under one coverslip area $(22 \times 50 \mathrm{~mm})$ were counted under low power (10× magnification) and recorded. If no microcrystal was seen on the first examination, the sample of bile stored at $37^{\circ} \mathrm{C}$ in the incubator was examined again after 24 hours. Crystals were considered to be absent only when they were not detected on two examinations - that is, immediately after collection and after 24 hours of incubation.

\section{CHEMICAL ANALYSIS OF BILE AND CALCULATION}

OF CSI

Both duodenal and gall bladder bile samples were subjected to chemical estimation for cholesterol, ${ }^{20}$ phospholipids, ${ }^{21}$ and total bile acids. $^{22}$ The CSI was calculated from Carey's table. ${ }^{23}$ Bile samples with a CSI of more than 1 were considered to be supersaturated.

\section{CHEMICAL ANALYSIS OF GALL STONES}

Gall stones were cut into two halves with a fine saw. One piece was finely powdered and used for chemical examination; the other piece was examined by scanning electron microscope (Philips SEM 515). The cholesterol content of gall stones was assayed by the chemical method described by From et al. ${ }^{20}$ Gall stones were classified as cholesterol stones if the total cholesterol content was more than $50 \%$ and as pigment stones if the cholesterol content was less than $20 \%$ of the dry weight of the stone. ${ }^{16}$ The cut as well as the external surface of each gall stone was coated with gold palladium in a vacuum and then subjected to scanning electron microscopy at magnification of $\times 2000$. Photographs were taken from the outer surface, and from the outer rim, middle, and central regions of the cut surface of each gall stone.

\section{STATISTICAL METHODS}

Statistical significance was calculated using $\chi^{2}$ test and McNemar's test. ${ }^{24}$

\section{Results}

CHEMICAL ANALYSIS OF GALL STONES

Of the 42 sets of gall stones, $38(90 \cdot 5 \%)$ were cholesterol stones, (cholesterol constituted a mean (SD) of 83.3 (9.4)\% of their dry weight) and four $(9.5 \%)$ were pigment gall stones (with a mean (SD) cholesterol content of $11 \cdot 5(7 \cdot 7) \%$ of the dry weight).

Scanning electron microscopy was done in all the 42 gall stones. The pigment stones $(n=4)$ consisted primarily of a uniformly amorphous structure. Twenty of 38 cholesterol stones, which predominantly showed rectangular cholesterol crystals, had clear cholesterol crystals on their outer surface (Fig 1): the remaining 18 had a shell of amorphous (pigment) material on their outer surface (Fig 2). Microspheroliths of calcium carbonate were present on the external 


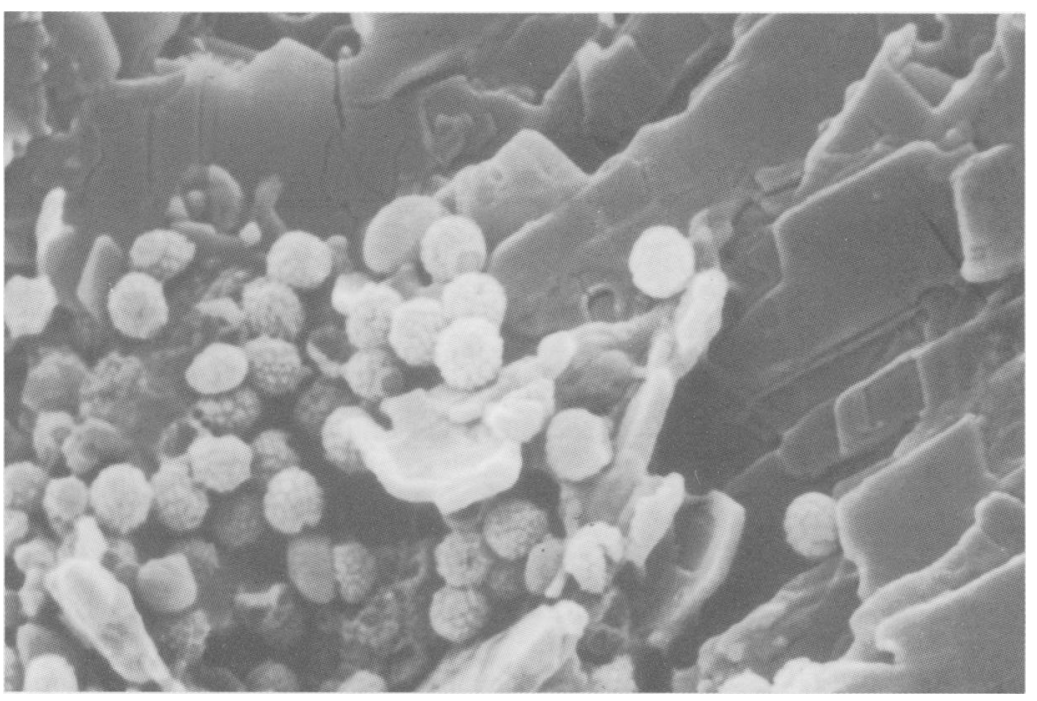

Figure 3: Scanning electron microphotograph (original magnification $\times 2000$ ) showing microspheroliths of calcium carbonate with underlying cholesterol crystals on the outer surface of the gall stone.
Calcium bilirubinate granules were detected in the duodenal bile of 27 patients. These were present alone in eight patients, four of whom had pigment stones (sensitivity $100 \%$, specificity $89 \%$ ) and in association with cholesterol crystals in 19 patients, 14 of whom had pigment shells around cholesterol stones. Calcium bilirubinate granules along with cholesterol monohydrate crystals were present in $14(78 \%)$ of the 18 patients who had cholesterol stones with pigment shells. In comparison they were seen in only eight of $20(40 \%)$ subjects who had cholesterol stones with clear surfaces $\left(\chi^{2}=4 \cdot 1\right.$, $\mathrm{p}<0.05)$. Therefore, the presence of calcium bilirubinate granules along with cholesterol crystals in duodenal bile was an indicator of pigment shells on cholesterol gall stones. In addition, cholesterol crystal counts were low $(<100$ per cover slip area) in 14 of 18 patients who had cholesterol stones with pigment shells compared with four of 20 who had cholesterol stones with clear surface $\left(\chi^{2}=10.4 ; \mathrm{p}<0.002\right.$, Fig 4).

Microspheroliths of calcium carbonate were detected in duodenal bile in three of six patients with calcium carbonate containing cholesterol gall stones (as detected by scanning electron microscopy) and showed a sensitivity of $50 \%$ and specificity of $100 \%$ for predicting the presence of calcium carbonate on outer layer of cholesterol gall stones. (10\%) were pigment stones, (b) six (14\%) were calcium carbonate containing cholesterol stones, and (c) 18 (43\%) were cholesterol stones enveloped by amorphous pigment shells. The remaining $14(33 \%)$ pure cholesterol stones could be considered to respond well to bile salt treatment.

MICROSCOPIC EXAMINATION OF DUODENAL BILE Satisfactory ' $\mathrm{B}$ ' bile samples were obtained in all 42 patients who completed the study. Cholesterol crystals were present in 34 of 38 (89\%) patients with cholesterol gall stones and were absent in all four patients with pigment gall stones. The presence of cholesterol crystals in duodenal bile had a sensitivity of $89 \%$ and specificity of $100 \%$ for predicting cholesterol gall stones.

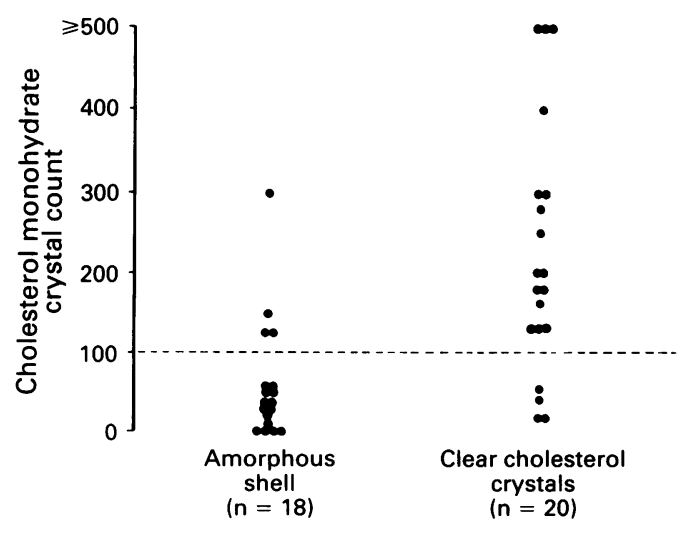

Gall stone surface

Figure 4: Relationship between the presence of an amorphous (pigment) shell and cholesterol crystal counts in duodenal bile $\left(\chi^{2}\right.$ test $\left.; p<0.002\right)$
CHEMICAL ANALYSIS OF DUODENAL BILE AND CSI Estimation of three biliary lipids - that is, cholesterol, phospholipids, and total bile acids, was performed in bile samples obtained from 39 patients, 35 of whom had cholesterol, and four pigment gall stones. Supersaturated bile (CSI $>1$ ) was present in 25 of $35(71 \%)$ patients with cholesterol gall stones and in none of the four patients with pigment gall stones. Supersaturated duodenal bile $($ CSI $>1)$ showed a sensitivity of $71 \%$ and specificity of $100 \%$ for predicting cholesterol gall stones.

MICROSCOPIC ANALYSIS OF GALL BLADDER BILE Cholesterol crystals were present in gall bladder bile in all 38 patients with cholesterol gall stones and were absent in all four patients with pigment gall stones, showing a sensitivity of $100 \%$ and specificity of $100 \%$ for predicting cholesterol gall stones. Calcium bilirubinate granules were present in gall bladder bile of 29 patients. They were present alone in all four patients with pigment stones (sensitivity of $100 \%$ and specificity of $100 \%$ for prediction of pigment gall stones); and in association with cholesterol crystals in other 25 patients with cholesterol stones. Calcium bilirubinate granules along with cholesterol crystals were present in 16 of $18(89 \%)$ cholesterol gall stones with pigment shells. Microspheroliths of calcium carbonate were present in the gall bladder bile in three of six patients with calcium carbonate containing cholesterol gall stones, giving a sensitivity of $50 \%$ and specificity of $100 \%$ for predicting calcium carbonate containing gall stones. 


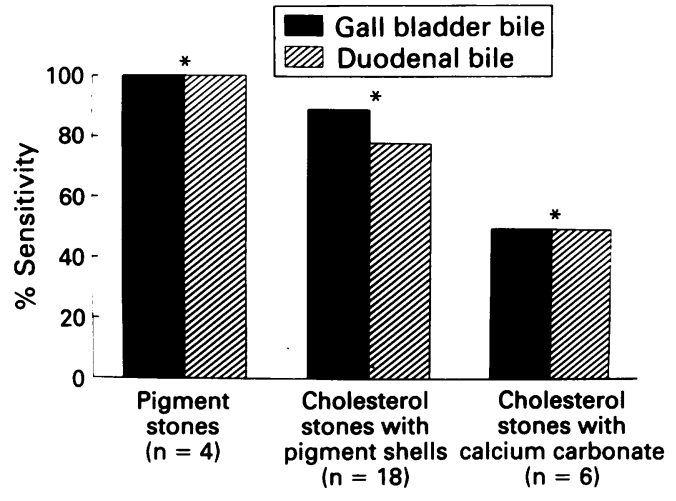

Figure 5: Comparison of results of microscopic examination of duodenal and gall bladder bile in identifying various subgroups of potential non-responders $(n=28)$ to bile salt treatment $\left({ }^{\star} p=N S\right)$.

CHEMICAL ANALYSIS OF GALL BLADDER BILE AND CSI

Thirty three (94\%) of 35 patients with cholesterol gall stones showed supersaturated (CSI >1) gall bladder bile. Bile samples of all four patients with pigment gall stones were unsaturated (CSI $<1)$. Supersaturated gall bladder bile showed a sensitivity of $94 \%$ and specificity of $100 \%$ for predicting cholesterol gall stones.

\section{COMPARISON OF DUODENAL BILE AND GALL}

\section{BLADDER BILE}

There was no significant qualitative difference in the microscopic examination findings of duodenal bile and gall bladder bile in their ability to predict cholesterol gall stones (34 (89\%) v 38 $(100 \%) ; \mathrm{p}=\mathrm{NS})$ and in predicting the potential non-responders to bile acid treatment (21 (75\%) $v 23(82 \%) ; \mathrm{p}=\mathrm{NS}$, Fig 5).

Of 35 patients with cholesterol gall stones, supersaturation (CSI $>1$ ) was present in 33 (94\%) and 25 (71\%) bile samples obtained from the gall bladder and duodenum respectively. This difference was statistically significant $(\mathrm{p}<0.05)$.

\section{COMPARISON OF MICROSCOPIC EXAMINATION} AND CSI

Of the two tests performed on duodenal bile samples, microscopic detection of cholesterol crystals (34 of 38 , sensitivity $89 \%$ ) was superior to CSI ( 25 of 35 , sensitivity $71 \%$ ) in predicting cholesterol gall stones $(\mathrm{p}<0.05)$. In gall bladder bile, however, there was no significant difference between the prediction rates of microscopic examination and CSI for cholesterol stones.

\section{Discussion}

Our study clearly shows that 28 of the 42 radiolucent gall stones could have been considered to resist dissolution with bile salt treatment as they were pigment stones, calcium carbonate containing cholesterol gall stones, and cholesterol stones with an amorphous (pigment) layer on the outer surface. Microscopic examination of duodenal bile identified $21(75 \%)$ of these potential non-responders. The results of bile salt therapy in patients shown to have radiolucent stones in a gall bladder in which filling is demonstrable on oral cholecystography have been poor: only 13 to $30 \%$ had complete dissolution. ${ }^{48}$ These criteria do not help distinguish those harbouring stones that are less likely to dissolve, many of which may be radiolucent. In view of the poor results of bile salt treatment, it is necessary to try and predict the composition of gall stones by simple in vivo tests so that stones which are unlikely to dissolve can be identified.

Our study, apart from being the largest prospective consecutive one to evaluate the utility of microscopic examination of duodenal bile in predicting gall stone composition, is the first to determine its role in the specific clinical context of a patient who fulfills the conventional criteria for oral bile acid therapy - that is radiolucent gall stones in a gall bladder that opacifies on oral cholecystography. In such a patient prediction of the chemical nature of the stones may be most important in either planning or prognosticating the outcome of dissolution therapy. Previous workers performed this test in a heterogenous group - that is in patients with undiagnosed abdominal pain for detecting microlithiasis ${ }^{17}$ or patients with ultrasonographically diagnosed gall stones in whom cystic duct patency had not been evaluated. ${ }^{16}{ }^{17}$ Their results were therefore at variance with ours, as some patients in their series had concomitant bile duct stones ${ }^{17}$ or might have had a cystic duct block that could have prevented good ' $B$ ' bile collection and could have influenced duodenal bile microscopy findings.

Although examination of bile obtained directly from the gall bladder has been shown to predict the chemical nature of gall stones quite accurately, ${ }^{1518}$ obtaining the bile sample from the gall bladder at surgery ${ }^{15}$ or by direct percutaneous puncture ${ }^{18}$ severely restricts its clinical usefulness, especially in patients who are being considered for non-invasive treatment of gall stones. Duodenal 'B' bile can be easily obtained with minimal discomfort or risk and may be of comparable diagnostic value. The next question we have therefore tried to answer is whether duodenal bile examination measures up in sensitivity to gall bladder bile. Our study shows that there was no statistically significant difference in the sensitivities of duodenal and gall bladder bile microscopic examinations in predicting cholesterol gall stones $(34(89 \%) v 38(100 \%)$; $\mathrm{p}=\mathrm{NS}$ ) or in identifying the potential nonresponders to bile salt treatment $(21(75 \%) v 23$ $(82 \%) ; \mathrm{p}=\mathrm{NS})$.

Using scanning electron microscopy, we have shown for the first time that amorphous shells envelop some cholesterol gall stones and prevent shedding of cholesterol crystals from the stone surface ${ }^{15}$; this is evidenced by absent or low cholesterol crystal counts and the concomitant presence of calcium bilirubinate granules in the bile of these patients. They can be prospectively identified by duodenal bile examination and excluded from oral bile acid therapy as they are known to fair poorly. ${ }^{1-3}$

The importance of calcium bilirubinate granules in bile for predicting pigment gall stones has not been well addressed in the published reports. When present alone, granules 
showed good sensitivity (100\%) for predicting pigment stones. The number of these stones was small, however, in our study because of their low occurrence in our population. Calcium bilirubinate granules, when present along with cholesterol crystals, may be derived from pigment shells around the cholesterol gall stones. Stasis and infection may lead to deposition of pigment on the surface of cholesterol stones. ${ }^{17}$

Various workers ${ }^{25} 26$ have found up to $50 \%$ of cholesterol gall stones to contain significant amounts of inorganic calcium, the carbonate salt being the most common. It is often present on the surface of the gall stone. We tried to correlate the presence of microspheroliths in bile with the presence of microspheroliths on the surface or the outer shell of the gall stone and noted that bile microscopy had a $100 \%$ specificity but a poor (50\% in both duodenal and gall bladder bile) sensitivity for detecting calcium carbonate in cholesterol gall stones rich in this compound. In the only other similar study Ros et $\mathrm{al}^{1}$ had shown similar specificity (100\%) and sensitivity (53\%) for their presence in duodenal bile and had shown them to be associated with a poor outcome on oral dissolution therapy. ${ }^{1}$ Therefore in spite of its limited sensitivity, detection of microspheroliths in bile is a sure indicator of calcium carbonate rich gall stones and would help in identifying the poor responders to oral bile acid treatment.

Previous studies ${ }^{15}$ with gall bladder bile had shown that microscopic detection of cholesterol crystals was a far better predictor of cholesterol gall stones than chemical estimation of biliary lipids. We have, for the first time, compared the sensitivity of the two tests in duodenal bile and shown that microscopic examination of duodenal bile is better than chemical estimation of biliary lipids (CSI) for prediction of gall stone composition.

A short nucleation time of the gall bladder bile has been shown to have a sensitivity of $78 \%$ and specificity of $100 \%$ for predicting cholesterol gall stones; it is not, however, superior to microscopic examination (cholesterol crystals). ${ }^{16}$ Furthermore, it is of no value in identifying cholesterol stones rich in calcium carbonate or those enveloped by an amorphous shell, and therefore has no role as a screening test for identifying potential non responders to bile acid treatment. Computed tomography of gall stones has also been evaluated for predicting gall stone composition. Low density stones are composed predominantly of cholesterol (good inverse relationship) but the correlation of increasing density value with calcium has been weak..$^{12}$ Pretreatment computed tomography to select patients with low density gall stones is an alternative approach that has been favourably reported. The failure rate has been still somewhat high $(36-50 \%) .{ }^{27} 28$

In conclusion, it seems reasonable to include microscopic examination of duodenal bile as a screening test for patients being considered for oral bile acid treatment to exclude potential nonresponders and thereby improve considerably the result of this treatment.
This work was supported by a grant from the intramural research fund of the Sanjay Gandhi Post-Graduate Institute of Medical Sciences, Lucknow.

1 Ros E, Navarro S, Fernandez I, Reixach M, Ribo JM, Rodes I. Utility of biliary microscopy for the prediction of chemical I. Utility of biliary microscopy for the prediction of chemical composition of gallstones and the outcome of dissolution therapy with

2 Freilich HS, Malet PF, Schwartz JS, Soloway RD. Chemica and morphologic characteristics of cholesterol gallstone hat failed to dissolve on chenodiol. The National Cooperative Gallstone Study. Gastroenterology 1986; 91: 713-8.

3 Whiting M, Jarvinen V, Watts J Mck. Chemical composition of gallstones resistant to dissolution therapy with chenodeoxycholic acid. Gut 1980; 21: 1077-81.

4 Schoenfield JL, Lachin JM, et al. Chenodiol (chenodeoxycholic acid) for dissolution of gallstones: the National deoxycholic acid) for dissolution of gallstones: the National and safety. Ann Intern Med 1981; 95: 257-82. Bachrach WH, Hofmann AF. Ursodeoxycholic acid in the treatment of cholesterol cholelithiasis. Dig Dis Sci 1982; 27: treatmen

6 Roda E, Bazzoli F, Morselli Labate AM, Mazzella G, Roda A Sama C, et al. Ursodeoxycholic acid vs chenodeoxycholic acid as cholesterol gallstone-dissolving agents: a comparative randomized study. Hepatology 1982; 2: 804-10.

7 Podda M, Zuin M, Battezzati PM, Ghezzi C, De Fazio C, Dioguardi ML. Efficacy and safety of a combination of chenodeoxycholic acid and ursodeoxycholic acid for gallstone dissolution: a comparison with ursodeoxycholic acid alone. Gastroenterology 1989; 96: 222-9.

8 Gleeson D, Ruppin DC, Saunders A, Murphy GM, Dowling RH. Final outcome of ursodeoxycholic acid treatment in 126 patients with radiolucent gallstones. $Q \mathcal{F}$ Med 1990; 279: patients

9 Trotman BW, Petrella EJ, Soloway RD, Sanchez H, Morris TA, Miller WR. Evaluation of radiographic lucency TA, Miller WR. Evaluation of radiographic lucency or opaqueness of gallstones as a means of identifying cholester

10 Bell GD, Dowling RH, Whitney B, Sutor DJ. The value of radiology in predicting gallstone type when selecting patients for medical treatment. Gut 1975; 16: 359-64

11 Dolgin SM, Schwartz JS, Kressel HY, Soloway Rd, Miller WT, Trotman BW, et al. Identification of patients with cholesterol or pigment stones by discriminant analysis of radiographic features. $N$ Engl f Med 1981; 304: 808-11.

12 Hickman MS, Sweisinger WH, Bora JD, Kurtin WE Computer tomographic analysis of gallstones. Arch Surg 1986; 121: 289-91.

13 Janowitz $P$, Zoller A, Swobodnik W, Wechsler JG, Schumacher KA, Ditschuneit $\mathrm{H}$. Computed evaluation of radiolucent gallstones in vivo. Gastrointest Radiol 1990; 15: $58-60$.

14 Brakel K, Lameris JS, Nijs HGT, Terpstra OT, Steen G, Blijenberg BC. Predicting gallstone composition with CT: in vivo and in vitro analysis. Radiology $1990 ; 174: 337-41$.

15 Ramond MJ, Dumont M, Belghiti J, Erlinger S. Sensitivity and specificity of microscopic examination of gall bladder bile for gall stone recognition and identification. Gastroenterology 1988; 5: 1339-43.

16 Erpecum VKJ, Van Berge-Henegouwen GP, Stoelwinder B Stolk MFJ, Egginck WF, Govaert WHA. Cholesterol an pigment gallstone disease comparison of the reliability of three bile test for differentiation between the two stone types. Scand f Gastroenterol 1988; 23: 948-54.

17 Delchier JC, Benfredj P, Preaux AM, Metreau JM Dhumeaux $D$. The usefulness of microscopic bile examination in patients with suspected microliothiasis: a prospective tion in patients with suspected microlioth. Hepatology 1986; 6: 118-22.

18 Janowitz P, Swobodnik W, Wechsler JG, Zoller A, Kuhn K, Ditschuneit $\mathrm{H}$. Comparison of gallbladder bile and endoscopically obtained duodenal bile. Gut 1990; 31: 1407-10.

19 Juniper K, Burson EN. Biliary tract studies. II. The significance of biliary crystals. Gastroenterology 1957; 32: 175-211.

20 Fromm H, Amin P, Klein H, Kupke I. Use of simple enzymatic assay for cholesterol assay. F Lipid Res 1980; 21 . 259-61.

21 Fiske $\mathrm{CH}$, SubbaRow Y. The colorimetric determination of phosphorus. F Biol Chem 1925; 66: 375-8.

22 Turley SD, Dietschy JM. Enzymatic analysis of bile acids F Lipid Res 1978; 19: 924-8.

23 Carey MC. Critical table for calculating the cholesterol saturation in native bile. $\mathcal{F}$ Lipid Res $1978 ; 19: 945-55$

24 Armitage $\mathrm{P}$, Berry G. Statistical methods in medical research. 2nd Ed. Cambridge, University Press, 1987: 120-2.

25 Sutor DJ, Wooley SE. The nature and incidence of gallstones containing calcium. Gut 1973; 14: 215-20.

26 Sutor DJ, Wooley SE. The sequential deposition of crystalline material in gallstones: evidence for changing gallbladder bile composition during the growth of some gallstones. Gut 1974 15: $130-1$

27 Walters JRF, Hood KA, Gleeson D, Ellul JPM, Keightley A Murphy GM, et al. Combination therapy with ora ursodeoxycholic acid and chenodeoxycholic acids: pretreatment computed tomography of the gall bladder improves gall stone dissolution efficacy. Gut 1992; 33: 375-80

28 Caroli A, Favero GD, Mario FD, Spigariol F, Scalon P Meggiato T, et al. Computed tomography in predicting gal stone solubility: a prospective trial. Gut 1992; 33: 698-700. 